\title{
Constraint of Complex Trace Analysis for Seismic Data Processing
}

\author{
S. M. Rahman ${ }^{*}$ \\ School of Physics, Universiti Sains Malaysia, 11800 Pulau Pinang, Malaysia
}

\begin{abstract}
Time frequency representation is a powerful tool for studying seismic reflection patterns and can thus provide useful information for stratification of the subsurface. Complex trace analysis, one of the geophysical techniques, is being employed for the time frequency analysis of seismic traces as analytic signal for the interpretation of seismic data. The applicability of the complex trace analysis in seismic data processing has been studied in this paper with few synthetic signals. The signals are analyzed with complex trace analysis for time frequency representations and compared with the spectral energy distributions. It is shown that complex trace analysis is not suitable for accurate estimation of time frequency representation of the signals having simultaneous frequencies.
\end{abstract}

Keywords: Time frequency; Complex trace; Analytic signal; Spectral analysis. (c) 2011 JSR Publications. ISSN: 2070-0237 (Print); 2070-0245 (Online). All rights reserved. doi:10.3329/jsr.v3i1.2106 J. Sci. Res. 3 (1), 65-73 (2011)

\section{Introduction}

Complex seismic trace analysis introduced by Taner [1] treats a seismic trace as the product of two independent and separable functions: instantaneous amplitude and cosine of the instantaneous phase. At any given time, instantaneous amplitude is the maximum value of the seismic trace and can attain under a constant phase rotation, and instantaneous phase is the phase angle required to rotate the trace to the maximum value [2]. Instantaneous amplitude and phase are the two basic seismic attributes and other attributes, e.g. frequency, relative amplitude change, wavelength, dip, azimuth etc. are derived from basic attributes through differentiation, averaging, combination, or transformation. Instantaneous frequency is a derived seismic attribute and a way of time frequency representation.

Time frequency representations of a signal can be made in many ways. The most straightforward approach would be to divide the signal into short time segments and determine the local spectrum by means of a Fourier transformation of the segment. The result of this operation is a widely used time frequency representation. The short-time or sliding-window Fourier transform of a signal inherently suffers from a trade-off between the length of the window and resolution of the representation.

\footnotetext{
*Corresponding author: smrahman@usm.my
} 
With the development of the concepts of time-frequency analysis, the number of publications on the subject has been steadily growing. In recent years, the theory of timefrequency analysis appears to have reached a more mature stage. However, there are many gaps and flaws in the theory that need to be addressed and the field is still developing rapidly. A comprehensive overview of the present state of affairs is given in two books by Cohen [3] and Flandrin [4]. The question of how to represent a signal as a joint function of time and frequency has been addressed by many researchers [5-10]. Most derivations of a fundamental time frequency representation start with the definition of a set of desirable properties that a time-frequency representation should have. This set of properties is then the starting point for the derivation of a representation that satisfies those properties. As a result, time-frequency analysis is made from different point of desired properties. SINFIT [11], AOK [12], STFT [13], zero-crossing, complex trace analysis [1] etc are examples of such methods of time frequency representations. This paper deals with the applicability of the complex trace analysis in seismic data processing. Rahman et al. [14-19] have shown the application of time frequency analysis in the interpretation of seismic reflection profiles using complex trace analysis. This trace analysis is a good technique. However it has some limitations for time frequency representation of a signal. Constraint of the technique is demonstrated in this research.

\section{Complex Trace Analysis}

Conventional seismic trace can be viewed as the real component of a complex trace which can uniquely be defined under usual conditions. The technique allows the separations of envelop amplitude and phase information, and the computation of instantaneous frequency [1]. Trace analysis treats a seismic trace, $y(t)$ as the real part of an analytic signal or complex trace, $Y(t)$. The complex trace can be written as [11, 15-19]:

$$
\begin{aligned}
Y(t) & =y_{r}(t)+j y_{i}(t) \\
& =y(t)+\frac{j}{\pi} \times \int \frac{s(\tau)}{t-\tau} d \tau \\
& =a(t) e^{j 2 \pi \phi(t)}
\end{aligned}
$$

where the imaginary part, $y_{i}(t)$, is given by the Hilbert transform as:

$$
y_{i}(t)=\frac{1}{\pi} \times \int \frac{s(\tau)}{t-\tau} d \tau
$$

The above integration implies taking the Cauchy principle value of the integral. The instantaneous amplitude and phase are

$$
a(t)=\sqrt{y_{r}^{2}(t)+y_{i}^{2}(t)}
$$


and

$$
\phi(t)=\tan ^{-1}\left(\frac{y_{i}(t)}{y_{r}(t)}\right)
$$

The instantaneous frequency, $f(t)$, is the rate of change of the time-dependent phase:

$$
f(t)=\frac{1}{2 \pi} \frac{d \phi(t)}{d t}
$$

\section{Analytical Signal}

Few analytic signals shown in Figs. 1 and 2 are drawn using most basic mathematical relations given in Eqs. (6) to (8):

$$
\begin{aligned}
& y(t)=a \sin (\omega t) \\
& y(t)=a_{1} \sin \left(\omega_{1} t\right)+a_{2} \sin \left(\omega_{2} t\right) \\
& y(t)=a_{1} \sin \left(\omega_{1} t\right)+a_{2} \sin \left(\omega_{2} t\right)+a_{3} \sin \left(\omega_{3} t\right)
\end{aligned}
$$

where, $a$ is amplitude coefficient, and $\omega$ is angular frequency and $\omega=2 \pi f t$.

In the first set (Fig. 1), there are three signals having respectively constant amplitude and frequency with time 0 to $1 \mathrm{sec}$ (Fig. 1(a)), variable amplitude and frequency in time 0 to 1 sec (Fig. 1(b)) and variable amplitude and frequency along with no signal (zero signal) (Fig. 1(c)) in time 0 to 1 sec.

a)

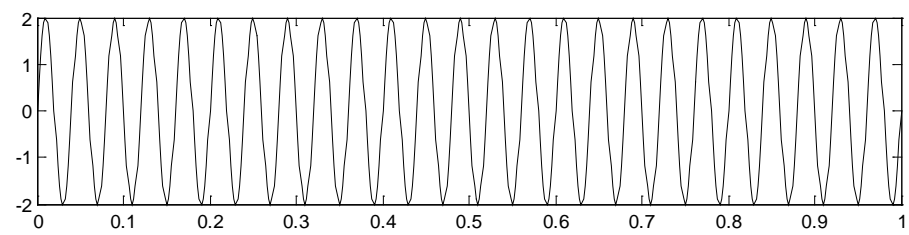

b)

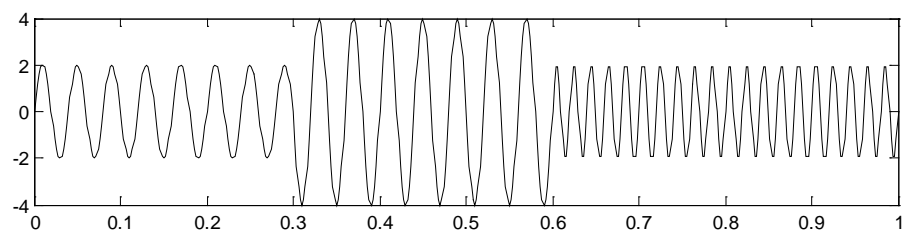

c)

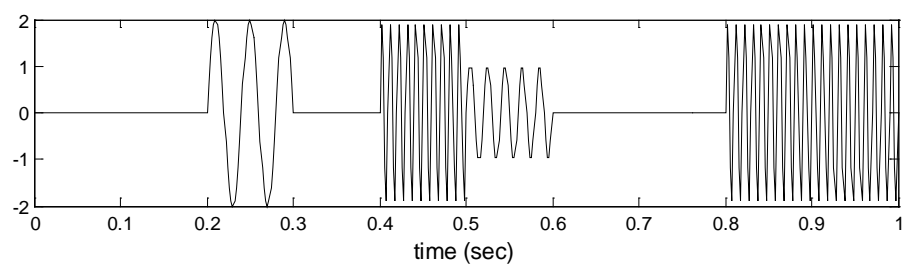

Fig. 1. Analytic signals, a) frequency $25 \mathrm{~Hz}$ (time span $0-1.0 \mathrm{sec}$ ), b) frequency $25 \mathrm{~Hz}$ in the time span 0-0.6 sec having higher amplitude in between 0.3-0.6 sec and frequency $50 \mathrm{~Hz}$ from 0.6-1.0 sec, c) frequency $25 \mathrm{~Hz}, 100 \mathrm{~Hz}, 50 \mathrm{~Hz}$ and $100 \mathrm{~Hz}$, respectively in the time span 0.2-0.3 sec., 0.40.5 sec., 0.5-0.6 sec. and 0.8-1.0 sec. 
In the second set (Fig. 2), with more complicated form, there are also three signals having respectively linear function of frequency with time as shown 0 to $1 \mathrm{sec}$ in Fig. 2(a), two simultaneous frequencies in the signal as shown 0 to $1 \mathrm{sec}$ in Fig. 2(b) and three simultaneous frequencies in the signal as shown 0 to $1 \mathrm{sec}$ in Fig. 2(c). Analytic signals are obtained from the computation of the simplified equations [Eqs. (6) to (8)].

a)

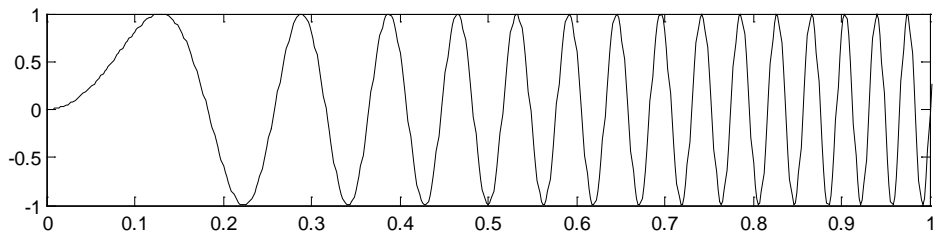

b)

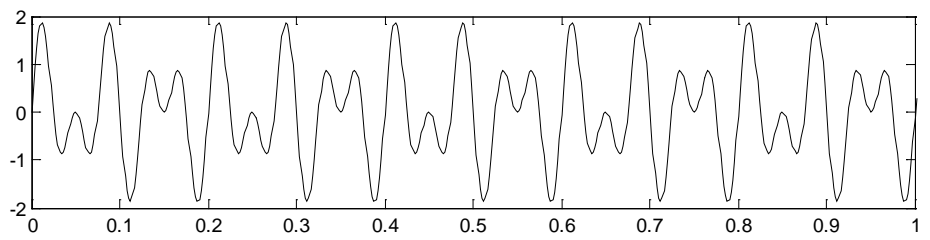

c)

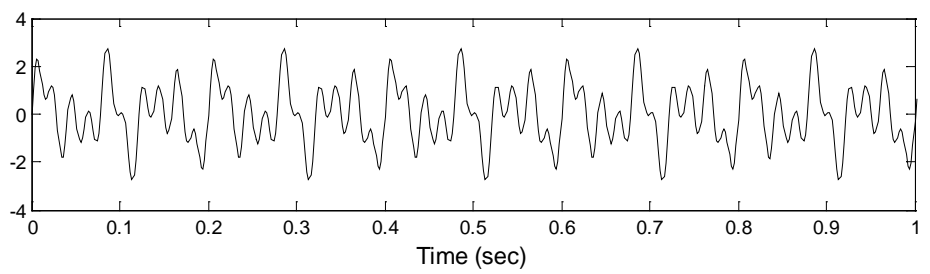

Fig. 2. Analytic signals, a) frequency is increasing linearly with time in $0-1 \mathrm{sec}$, b) two frequency components $(15 \mathrm{~Hz}$ and $25 \mathrm{~Hz}$ ) present at the same time in 0 to $1 \mathrm{sec}$, and c) three frequency components $(15 \mathrm{~Hz}, 25 \mathrm{~Hz}$ and $50 \mathrm{~Hz})$ present at the same time in 0 to $1 \mathrm{sec}$.

\section{Spectral Analysis}

The spectral analysis is a procedure that decomposes a time series into a spectrum of cycles of different lengths. It is also known as frequency domain analysis. The spectral analysis describes the distribution of the power at a specific frequency of a signal, based on a finite set of data. The estimation of power spectra is useful in many applications, including the detection of signals from the noises. In the present article, the power spectra of the analytical signals (Figs. 1 and 2) are made with discrete-time Fourier transform. The power spectra of the analytic signals are shown in Figs. 3 and 4.

\section{Time Frequency Analysis}

The application of time frequency analysis in seismic processing is particularly used to see the local effect of the seismic trace, by which interpreter can assume the geologic features from the analysis. Complex trace analysis, one of the time frequency analyses in geophysical technique, is an attractive tool in many occasions. Employing complex trace analysis or using Eq. (5), time frequency representations of the analytic signals (Figs. 1 
and 2) are estimated and shown in Figs. 5 and 6. The obtained results and constraints of the technique are discussed in the following section.

\section{Results and Discussions}

In this article, the spectral analysis and time frequency analysis of few synthetic signals are estimated. Spectral analysis using Fourier transformation is thought to be the most fundamental and more acceptable analysis in any signal processing operation. In the present article, power spectrum made with Fourier transform is also considered as the prime analysis. On other hand, estimated time frequency analyses with complex trace analysis are compared with power spectra of the analytic signals.

Figs. 3(a-c) show the power spectra of the three analytic signals as shown in Figs. 1(ac) respectively with constant amplitude and frequency $(25 \mathrm{~Hz})$, variable amplitude and frequency ( $25 \mathrm{~Hz}$ and $50 \mathrm{~Hz}$ ), and variable amplitude and frequency (25 Hz, $100 \mathrm{~Hz}$ and $50 \mathrm{~Hz}$ ) along with zero signal (no signal). From the analyses it has seen that the highest spectral energy densities are obtained respectively at $25 \mathrm{~Hz}, 25 \mathrm{~Hz}$ and $50 \mathrm{~Hz}$, and $25 \mathrm{~Hz}$, $50 \mathrm{~Hz}$ and $100 \mathrm{~Hz}$ as shown in Figs. 3(a-c). Analyses are well satisfied with the variables (amplitude and frequency) used in analytic signals (Fig. 1). Time frequency analyses of the same analytical signals (Fig. 1) with complex trace analysis are shown in Figs. 5(a-c). These analyses are also satisfied with the variables used in analytic signals. Both the power spectra with Fourier transformation (Fig. 3) and the time frequency analyses with complex trace analysis (Fig. 5) lead to an estimation of accurate signal variables.

a)

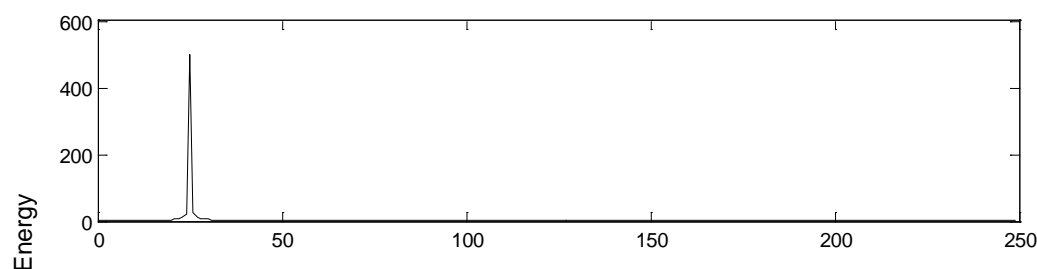

b)

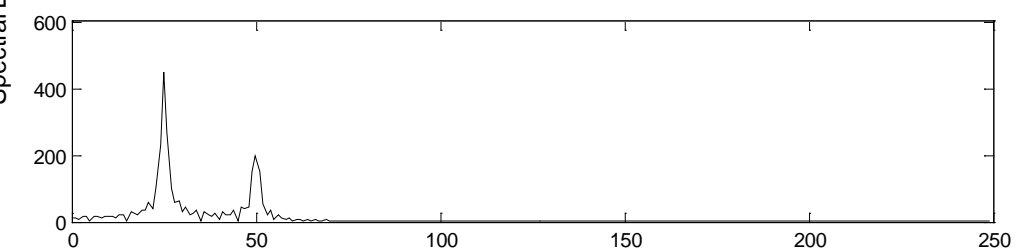

c)

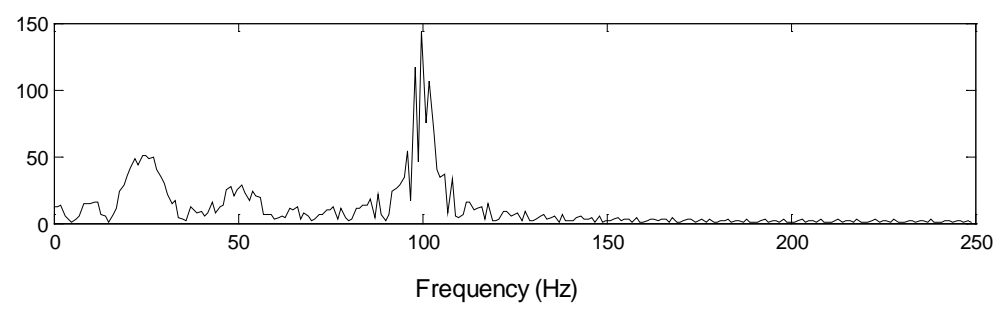

Fig. 3. Power spectra of the corresponding 3 analytical signals shown in Fig. 1. 
a)

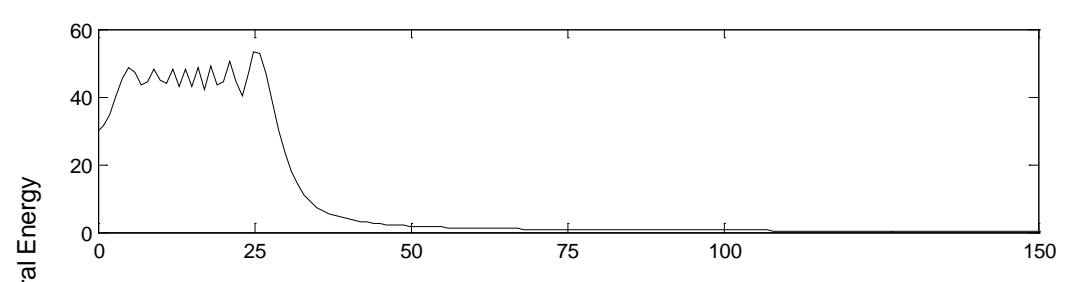

b)

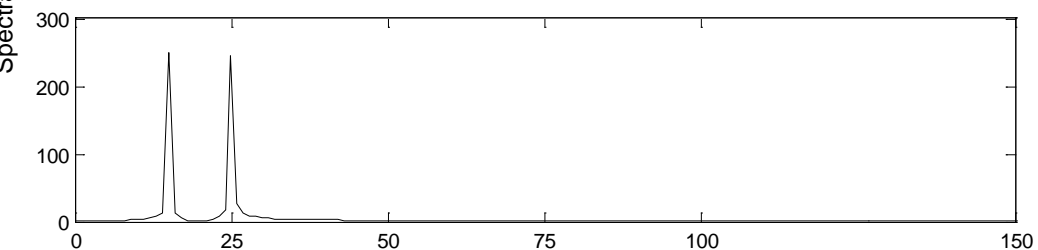

c)

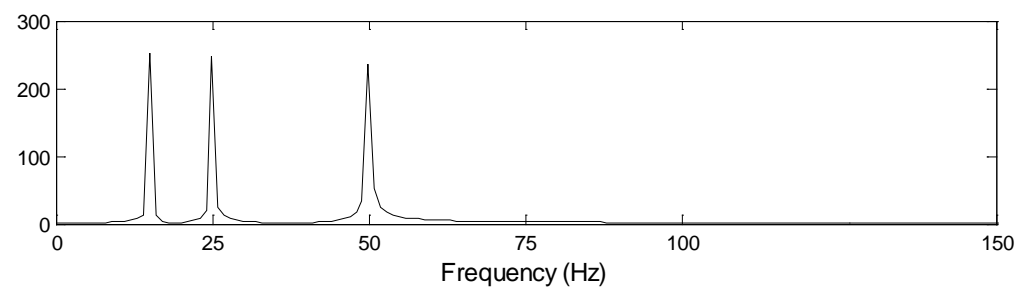

Fig. 4. Power spectra of the corresponding analytic signals shown in Fig. 2.
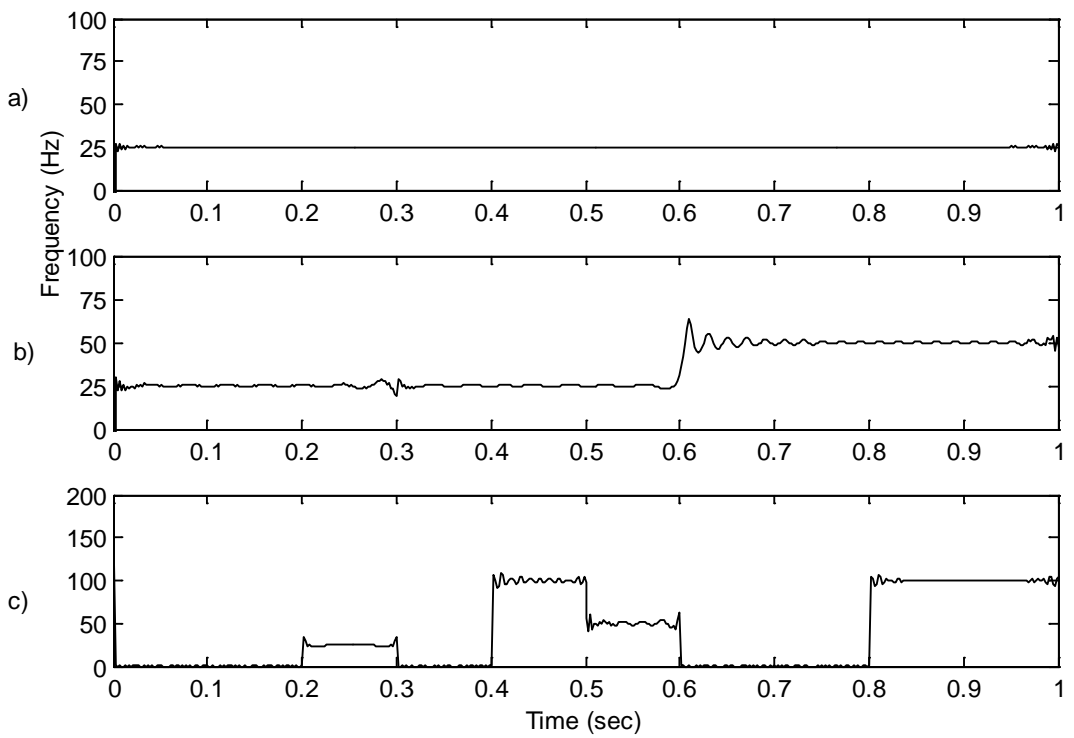

Fig. 5. Time frequency analysis of the corresponding analytic signals shown in Fig. 1 with complex trace analysis. 
a)

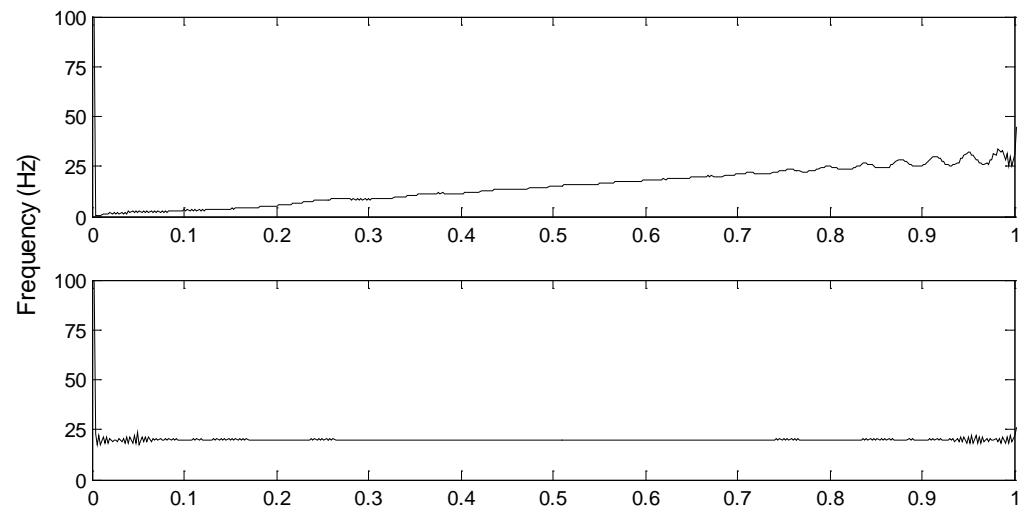

c)

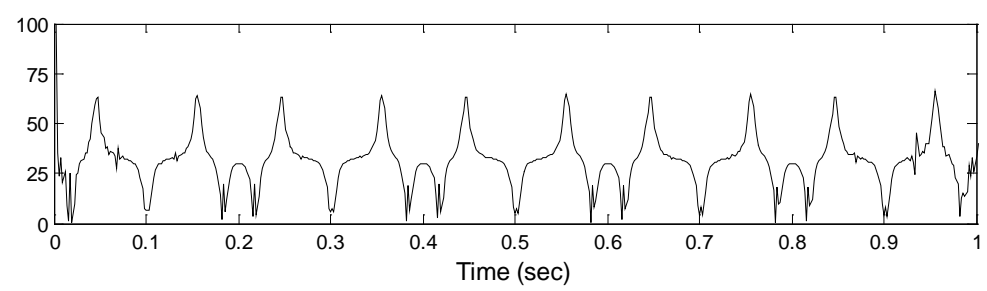

Fig. 6. Time frequency analysis of the corresponding analytic signals shown in Fig. 2 with complex trace analysis.

Similarly the power spectra and time frequency analyses are shown in Fig. 4 and Fig. 6, respectively of another set of analytic signals as shown in Figs. 2(a-c). Here, the time frequency analyses are not the same as those found in spectral analyses except the signal in which frequency varies linearly with time (Fig. 2(a)). Power spectra (Figs. 4(a-c)) have enabled estimation of accurate signal variables used in the analytic signals. But complex trace analyses (Figs. 6(b-c)) have not led to accurate signal variables in the time frequency representations. Figs. 2(b-c) are the analytic signals having two and three simultaneous frequencies and these are implemented using Eqs. (7) and (8). Power spectra, as shown in Figs. 5(b-c), lead to correct estimations of two $(15 \mathrm{~Hz}$ and $25 \mathrm{~Hz})$ and three $(15 \mathrm{~Hz}, 25 \mathrm{~Hz}$ and $50 \mathrm{~Hz}$ ) frequencies of the signals. However, complex trace analyses have yielded one frequency (instead of two frequencies) of $20 \mathrm{~Hz}$, which seems to be the average value of $15 \mathrm{~Hz}$ and $25 \mathrm{~Hz}$ of the analytic signal (Fig. 2(b)), and a range of frequencies (instead of three separate frequencies) varying between 0-63 Hz (Fig. 6(c)), which is also not the same as the frequencies used in the analytic signal (Fig. 2(c)) of $15 \mathrm{~Hz}, 25 \mathrm{~Hz}$ and $50 \mathrm{~Hz}$. Therefore complex trace analysis is not suitable for time frequency analysis of the signals having multiple simultaneous frequencies.

Time frequency analysis with complex trace analysis has shown a suitable technique for the analytic signals (Figs. 1(a-c) and Fig. 2(a)). Complex trace analysis has led to almost accurate instantaneous frequency as shown in Figs. 5(a-c) and Fig. 6(a) as time frequency analysis. Little variations are also observed at the beginning and at end of the 
analytic signals using trace analysis but the technique is quite unique. On other hand, the time frequency analyses shown in Figs. 6(b-c) of the analytical signals (Figs. 2(b-c)) are not found accurate estimations of instantaneous frequency. Therefore complex trace analysis might not be the better approach for the analytic signal having two or more simultaneous frequencies. It is observed that from the point of computational cost and for monochromatic analytic signal in which frequency of the signal can be varied with time (one frequency component in each time), complex trace analysis is a very good tool for time frequency analysis. However, complex trace analysis is not suitable for the time frequency analysis of the signals having two or more frequency components at the same time. In most of the practical cases the detected seismic signals might be the combination of multiple simultaneous frequencies as various effects caused by subsurface; hence complex trace analysis may not be the right choice on that occasion for the estimation of time frequency representations.

\section{Conclusion}

Complex trace analysis is a good technique for time frequency analysis of the signals having only one frequency at a time. However, practically the detected seismic signal might be the resultant effect of multiple simultaneous frequencies or the combination of several other signals with source signal. This work has demonstrated that the complex trace analysis cannot estimate accurate time frequency representations of the signals having multiple frequencies at the same time. For the signal having two simultaneous frequencies, complex trace analysis estimates an average value, which is not correct. Similarly for the signal having more than two simultaneous frequencies, complex trace analysis estimates a range of frequencies instead of accurate frequencies. It is thus concluded that the complex trace analysis cannot provide accurate time frequency representation of the signals having multiple simultaneous frequencies.

\section{References}

1. M. T. Taner, F. Koehler, and R. E. Sheriff, Geophysics 44 (6), 1041 (1979). doi:10.1190/1.1440994

2. A. E. Barnes, Geophysics 72, W33 (2007). doi:10.1190/1.2785048

3. L. Cohen, Time-Frequency Analysis (Prentice Hall Signal Processing Series, New Jersey, 1995).

4. P. Flandrin, In: Temps-Fréquence (Hermès, Paris, 1993).

5. D. Gabor, J. IEE (London) 93, 26 (1946).

6. J. Ville, Cables et Trans-missions 2A, 1 (1948).

7. C. H. Page, Journal of Applied Physics 23, (1952).

8. M. B. Priestley, J. Royal Stat. Soc. Series B 27, (1965).

9. A. Rihaczek, IEEE Trans. on Information Theory 14, 3 (1968). doi:10.1109/TIT.1968.1054157

10. W. E. Mark, J. Sound Vib. 11, 19 (1970).

11. H. H. Hardy, R. A. Beier, and J. D. Gaston, Geophysics 68, 1 (2003). doi:10.1190/1.1543222

12. P. Steeghs and G. Drijkoningen, Geophysics 66, 6 (2001). doi:10.1190/1.1487136

13. A. Chakraborty and D. Okaya, Geophysics 60, 6 (1995). doi:10.1190/1.1443922 
14. S. M. Rahman, M. R. Islam, M. Keramat., M. M. Alam, and M. Kimura, Bull. Faculty Sc. Univ. Ryukyus 80, 9 (2005).

15. S. M. Rahman, Ph.D. Thesis, University of Rajshahi, Bangladesh (2006).

16. S. M. Rahman, M. R. Islam., M. Keramat, and M. S. Islam, J. Geol. Soc. India 69, 4 (2007).

17. M. A. Rahman, S. M. Rahman, M. R. Islam, and M. Keramat, J. Geol. Soc. India 71, 5 (2008).

18. S. M. Rahman, M. R. Islam, M. Keramat, and M. S. Islam, Icfai Univ. J. Earth Sci. 2, 4 (2008).

19. S. M. Rahman, M. R. Islam., M. Keramat, and M. S. Islam, Earth Sci. Res. J. 12, 2 (2008). 